\title{
Rückläufige Verkehrsmengen?!
}

\author{
Welche Konsequenzen zieht die Regionalplanung für ihr funktionales Straßennetz? \\ Das Beispiel Mittelthüringen
}

\section{Falling Traffic Volumens?}

What consequences should regional planning draw for its functional road network? A case-study from Central Thuringia

\section{Kurzfassung}

Der Beitrag untersucht, inwiefern das raumordnerische Instrument der Funktionalen Straßennetze unter den Vorzeichen des demographischen Wandels neu justiert werden muss. Anhand der Planungsregion Mittelthüringen wird zunächst beispielhaft analysiert, ob Schrumpfung und Überalterung der Bevölkerung auf die Verkehrsmengen durchschlagen oder aber von anderen Faktoren kompensiert werden. Dabei zeigt sich, dass der Straßenverkehr tatsächlich nicht mehr grenzenlos wächst, sondern vielmehr in manchen Gebieten zwischen 1995 und 2000 schon rückläufig war. Für die Zukunft zeichnet sich ein weiteres Wachstum im Wesentlichen nur noch für dieVerbindungen mit europäischer Bedeutung ab, während vor allem imi dünn besiedelten ländlichen Raum mit einer Abnahme der Verkehrsmengen zu rechnen ist. Für die Autoren leitet sich unter den gegebenen demographischen und finanziellen Bedingungen daraus ab, dass die Regionalplanung künftig nicht mehr im großen Stil Ausbaumaßnahmen für alle Kategorien des Funktionalen Straßennetzes fordern kann und sollte (so wie das bislang in Mittelthüringen der Fall war), sondern sich mit Fragen der Verkehrsmengenentwicklung auseinandersetzen muss.

\begin{abstract}
This article considers the extent to which it is now necessary to make adjustments to socalled "functional road networks", an instrument of comprehensive spatial planning, in response to changes in demographics. Drawing on a case-study of the Central Thuringia planning region, the analysis focuses initially on the question of whether population shrinkage and ageing actually impact on levels of road traffic, or whether their effects are offset by other factors. As the authors report, road-traffic volumes are no longer growing relentlessly; in the period between 1995 and 2000, they were, in some areas, already in decline. Additional growth in the future is likely, however, essentially on those routes which are of European significance, whilst the more sparsely populated rural regions are expected to see a continuing decline in traffic levels. The conclusion drawn by the authors in the light of prevailing demographic and financial circumstances is that regional planning can - and should - no longer go on calling for the expansion of all categories of the functional road network on a large scale (as has previously happened in Central Thuringia); it should rather address the issues thrown up by the actual development of traffic volumes.
\end{abstract}




\section{Einleitung}

Der demographische Wandel ist derzeit - zumindest in den bereits vom Bevölkerungsrückgang betroffenen Gebieten - wohl eines der meist diskutierten Themen in der Raumordnung: Debattiert wird über das Ausmaß der Veränderungen und vor allem über die Folgen für die soziale Infrastruktur sowie über die daraus abzuleitenden Konsequenzen für das Zentrale-Orte-System. Deutlich weniger Aufmerksamkeit wird dagegen dem Verkehr gewidmet. Während jedoch schon vereinzelt untersucht wurde, welche Auswirkungen der demographische Wandel auf den öffentlichen Verkehr haben wird und welche Handlungsoptionen für den öffentlichen Nahverkehr verbleiben ${ }^{1}$, ist die Frage der Gestaltung des Straßennetzes unter den Bedingungen von Bevölkerungsrückgang und Überalterung bislang kaum betrachtet worden.

Für jeden Planer in einer Region mit rückläufiger Bevölkerungsentwicklung wirft sich im Rahmen der Fortschreibung eines Raumordnungsplans heute die Frage auf, wie mit den zu erwartenden demographischen Entwicklungen in Bezug auf die Funktionalen Verkehrsnetze umzugehen ist. Insbesondere stellt sich die Frage, inwieweit die Verkehrsmengenentwicklung dem absehbaren Bevölkerungsrückgang folgen wird. Und sollte ein Absinken der Verkehrsmengen zu erwarten sein: Inwiefern sollte dies Auswirkungen auf die Gestaltung der Funktionalnetze haben, die sich ja grundsätzlich nicht an Verkehrsstärken orientieren, sondern aus der Verbindung zwischen Zentralen Orten bzw. der Anbindung Zentraler Orte an übergeordnete Funktionalnetzebenen begründen?

Im Folgenden soll versucht werden, am Beispiel der Region Mittelthüringen, wo das Verfahren zur Fortschreibung des Regionalen Raumordnungsplans bereits eingeleitet wurde, diese Fragen bezogen auf das Funktionale Straßennetz zu beantworten.

\section{Ausgangssituation in Mittelthüringen}

Für die Region Mittelthüringen besteht seit 1999 ein kompletter verbindlicher Regionalplan ${ }^{2}$, für den im Juni 2004 das Fortschreibungsverfahren eingeleitet worden ist.

Kernstücke der zukünftigen Festsetzungen im Bereich Verkehr werden - wie bereits im gültigen Regionalplan von 1999 - die funktionalen Netze sein. Das Funktionale Straßennetz entfaltet seine Steuerungswirkung vor allem

- beim Ausbau und Neubau von Straßen(abschnitten), indem es zur Ermittlung der Verbindungsfunktionsstufe nach der RAS- $\mathrm{N}^{3}$ herangezogen wird, die wie- derum maßgeblichen Einfluss auf alle Trassierungsparameter hat,

- als Maßstab für die Gewichtung verkehrlicher Belange in der Abwägung mit anderen Belangen im Rahmen von Raumordnungsverfahren zu Straßenplanungen sowie

- durch ergänzende Festsetzungen zur Gestaltung des Straßennetzes, sowohl allgemeiner Art für die Ebenen des Funktionalnetzes als auch spezieller Art für einzelne Maßnahmen, die ebenfalls im Rahmen der Raumordnungsverfahren Berücksichtigung finden.

Der Landesentwicklungsplan Thüringen 2004 (LEP) $^{4}$ gibt für die Funktionalnetze die Verbindungskategorien vor. Gemäß Ziel 4.1.6 LEP werden sie vierfach unterteilt in ein europäisch, großräumig, überregional und regional bedeutsames Verkehrsnetz.

Aufgabe der Regionalplanung ist es nunmehr, erstens die im LEP bereits abschließend zeichnerisch festgelegten Verbindungen der ersten drei Kategorien durch präzisierende und ergänzende Zielstellungen zu untersetzen und zweitens die regionalen Verbindungen als vierte Ebene zeichnerisch und textlich zu definieren

\section{Entwicklung der Straßenverkehrsmengen in Mittelthüringen 1995 bis 2000}

\subsection{Methodik und Hypothesen}

Methodisch greift die Analyse der Verkehrsmengenentwicklung auf zwei Verkehrsmengenkarten vom Thüringer Landesamt für Straßenbau für die Jahre 1995 und 2000 zurück. Sie enthalten für Zählstellen an den Autobahnen, Bundes- und Landesstraßen Daten zum durchschnittlichen täglichen Kfz-Verkehr und zum durchschnittlichen täglichen Schwerlastverkehr $>3,5 \mathrm{t}^{5}$ Insgesamt wurden die Daten von 162 Zählstellen, die 1995 und 2000 von ihrer Lage her unverändert geblieben sind, ausgewertet. Betont werden muss, dass sich alle Analyseergebnisse naturgemäß nur auf die Verkehrsmengen an den Zählstellen beziehen, also nicht die tatsächliche Gesamtverkehrsmenge in Mittelthüringen erfasst wird.

Unter Berücksichtigung der wichtigsten Rahmenbedingungen werden folgende Hypothesen für die Verkehrsmengenentwicklung in Mittelthüringen zugrunde gelegt:

(1) Der motorisierte Individualverkehr (MIV) dürfte zwischen 1995 und 2000 insgesamt gewachsen sein, vor allem jedoch in der Fläche. Zwar ist einerseits die Bevölkerung zurückgegangen, andererseits wurde aber die Siedlungsstruktur disperser, während gleichzeitig 
Tabelle 1

Verkehrsmengen nach Einordnung im Funktionalnetz (LEP Thüringen 2004) in 1000 Fahrzeuge (DTV) und prozentuale Veränderung zwischen $1995-2000^{6}$

\begin{tabular}{|l|r|r|r|r|r|r|r|r|r|}
\hline \multirow{2}{*}{$\begin{array}{l}\text { Verbindung mit ... } \\
\text { Bedeutung }\end{array}$} & \multicolumn{3}{|c|}{ MIV } & \multicolumn{3}{c|}{ Schwerlastverkehr } & \multicolumn{4}{c|}{ Gesamtverkehr } \\
\cline { 2 - 12 } & $\mathbf{1 9 9 5}$ & $\mathbf{2 0 0 0}$ & $\mathbf{\%}$ & $\mathbf{1 9 9 5}$ & $\mathbf{2 0 0 0}$ & $\mathbf{\%}$ & $\mathbf{1 9 9 5}$ & $\mathbf{2 0 0 0}$ & $\%$ \\
\hline europäisch & 379 & 452 & +16 & 79 & 102 & +22 & 459 & 554 & +17 \\
\hline großräumig & 83 & 77 & -9 & 9 & 8 & -23 & 93 & 85 & -10 \\
\hline überregional & 257 & 296 & +13 & 31 & 27 & -14 & 288 & 323 & +11 \\
\hline regional & 187 & 225 & +17 & 17 & 15 & -14 & 204 & 241 & +15 \\
\hline Keine (außerorts) & 236 & 280 & +16 & 24 & 17 & -36 & 260 & 297 & +13 \\
\hline Keine (innerorts) & 58 & 54 & -8 & 4 & 3 & -58 & 63 & 57 & -10 \\
\hline Gesamt & $\mathbf{1 2 0 2}$ & $\mathbf{1 3 8 4}$ & $+\mathbf{1 3}$ & $\mathbf{1 6 6}$ & $\mathbf{1 7 2}$ & $+\mathbf{+ 4}$ & $\mathbf{1 3 6 7}$ & $\mathbf{1 5 5}$ & $+\mathbf{5 5}$ \\
\hline
\end{tabular}

beispielsweise Bahnnebenstrecken stillgelegt wurden und parallel der Pkw-Besatz angestiegen ist.

(2) Im Bereich des Schwerlastverkehrs dürfte vor allem die wirtschaftliche Entwicklung maßgebend gewesen sein. Deutliche Zunahmen beim Bruttoinlandsprodukt, speziell bei den Im- und Exporten, lassen vermuten, dass der Schwerlastverkehr insgesamt in diesem Zeitraum angestiegen ist, insbesondere aber auf den europäischen / großräumigen Verbindungen Verkehrsmengenzuwächse durch arbeitsteilige Produktionsprozesse zu verzeichnen waren.

\subsection{Verkehrsmengenentwicklung 1995 bis 2000}

\section{Gesamtverkehr}

Der an den Zählstellen ermittelte Verkehr hat um $12 \%$ zugenommen. Dabei haben besonders die Bundesautobahnen (Straßen mit europäischer Bedeutung) und die Landesstraßen (im Wesentlichen regionale Verbindungen) eine überdurchschnittliche Zunahme von $16-17 \%$ zu verzeichnen. Dagegen gab es zwischen 1995 und 2000 einen unterdurchschnittlichen Zuwachs der Verkehrsmengen auf den Bundesstraßen in Mittelthüringen. Besonders die B4 Erfurt - Nordhausen als großräumige Verbindung hatte Rückgänge zu verzeichnen, was mit signifikanten Abnahmen der Pendlerverflechtungen zwischen Nord- und Mittelthüringen begründet werden kann. ${ }^{7}$

\section{Schwerlastverkehr und Schwerlastverkehrsanteil}

Die Entwicklung des Schwerlastverkehrs zeigt bei moderater Zunahme von $4 \%$ binnen eines Zeitraums von fünf Jahren ein ambivalentes Bild. Auf der einen Seite ist eine starke Zunahme auf europäisch bedeutsamen Straßenverbindungen zu verzeichnen, auf der anderen Seite zeigen sich starke Abnahmen in den anderen $\mathrm{Ka}$ tegorien ,des Funktionalnetzes. Noch stärker zurückgegangen ist der Schwerlastverkehr auf den Straßen ohne funktionale Kategorisierung (um durchschnittlich $36 \%$ ). Räumlich gesehen ergibt sich damit eine Konzentration auf die Straßen des Funktionalnetzes, hauptsächlich auf die Verbindungen mit europäischer Bedeutung.

Augenfällig beim Schwerlastverkehr ist allerdings eine relativ starke Streuung unterhalb der großräumigen Verbindungen (s. Tab. 2). Sie kommt dadurch zustande, dass einige Verbindungen teilweise aufgrund von Ausbaumaßnahmen, teilweise bedingt durch Sondersituationen wie Baustellenverkehr hohe Zuwächse zu verzeichnen hatten. Demgegenüber scheinen die Kategorien des Funktionalnetzes im MIV relativ homogen zu sein.

Tabelle 2

Streuung der Entwicklungswerte in den Kategorien des Funktionalnetzes ${ }^{8}$

\begin{tabular}{|l|c|c|}
\hline Straßenkategorie & $\begin{array}{l}\text { Standard- } \\
\text { abweichung } \\
\text { MIV }\end{array}$ & $\begin{array}{l}\text { Standard- } \\
\text { abweichung } \\
\text { Schwerlastverkehr }\end{array}$ \\
\hline europäisch & 16 & 17 \\
\hline großräumig & 20 & 19 \\
\hline überregional & 22 & 43 \\
\hline regional & 28 & 92 \\
\hline Keine (außerorts) & 28 & 49 \\
\hline Keine (innerorts) & 41 & 93 \\
\hline
\end{tabular}


Der Schwerlastanteil auf den einzelnen Straßenkategorien des Funktionalnetzes verteilt sich wie folgt: $18 \%$ des Verkehrs auf europäisch bedeutsamen Straßen (Autobahnen) sind Lkws, bei den restlichen Kategorien wird ein deutlich geringerer Anteil erreicht (8-9\%), und Straßen ohne Kategorisierung im Funktionalnetz (außerorts) haben einen Schwerlastanteil von nur 5-6\%. Im Ganzen ist der Anteil leicht rückläufig (1995: $12 \%$, 2000: $11 \%$ ), auf den gezählten Autobahnabschnitten zeigt sich aber ein leichter Anstieg.

Insgesamt gesehen spiegelt das neue Funktionalnetz des Regionalplans daher sowohl die Schwerlastverkehrsmengen als auch den Schwerlastverkehrsanteil wider. Das Funktionalnetz erweist sich als steuerungswirksam im Sinne der Aufnahme eines Großteils dieses Verkehrs.

Die oben formulierte These zum Schwerlastverkehr lässt sich dagegen nicht im vollen Maße bestätigen. Zwar gab es in Korrelation zum Wirtschaftswachstum eine Steigerung. Was jedoch dem allgemein unterstellten Zusammenhang zwischen wirtschaftlicher Entwicklung und Verkehrsmengenzunahme widerspricht, ist der stark zurückgegangene Schwerlastverkehr auf allen Straßenkategorien unterhalb der europäisch bedeutsamen Verbindungen. Es kann nachgewiesen werden, dass insbesondere der Transitverkehr entlang der A4 zu den Zuwächsen beigetragen hat. Die überproportional gestiegenen Exportumsätze können ebenfalls das Wachstum der Verkehrsmenge begründen, wenngleich offen bleibt, warum nur die BAB von diesem Trend profitierte. Das wirtschaftliche Wachstum einer Region kann daher offensichtlich nicht per se als Basisannahme für Verkehrsmengenprognosen genutzt werden.

\section{Motorisierter Individualverkehr}

Die Entwicklung des motorisierten Individualverkehrs (MIV) liegt leicht über dem Durchschnitt der Gesamtverkehrsmenge. Dabei sind die Wachstumsraten der Verbindungen mit europäischer und regionaler Bedeutung beachtenswert, hinzukommen die Straßen ohne Kategorisierung im Funktionalnetz. Die oben aufgestellte These zum MIV bestätigt sich somit: Während großräumige und überregionale Straßenverbindungen Abnahmen bzw. unterdurchschnittliches Wachstum zeigen, scheint sich der Verkehr auf regionale Straßenverbindungen bzw. in die Fläche zu verlagern. Zwar hat der Verkehr auf der A4 insgesamt gesehen zugenommen, aber er hat sich auf den einzelnen Streckenabschnitten sehr unterschiedlich entwickelt, war zum Teil sogar rückläufig. Im Rückschluss lässt sich daraus ableiten, dass die Veränderungen der Verkehrsmengen beim MIV auf den Autobahnabschnitten im Wesentli- chen aus dem regionalen Verkehr und nicht aus dem Transit herrühren. Eine starke Zunahme auf der A4 ist zwischen Gotha und Erfurt-West zu verzeichnen, bedingt durch geänderte Verkehrsströme im Zuge des Neubaus der A71. Wird dieser Sondereffekt herausgerechnet, ergibt sich für die BAB 4 als Straßenverbindung von europäischer Bedeutung keine überdurchschnittliche Zunahme. Hier bestätigt sich die oben formulierte These zum MIV ebenfalls.

\section{Abbildung 1 \\ Schematische Darstellung der Verkehrsmengenentwicklung in Mittelthüringen 1995-2000}

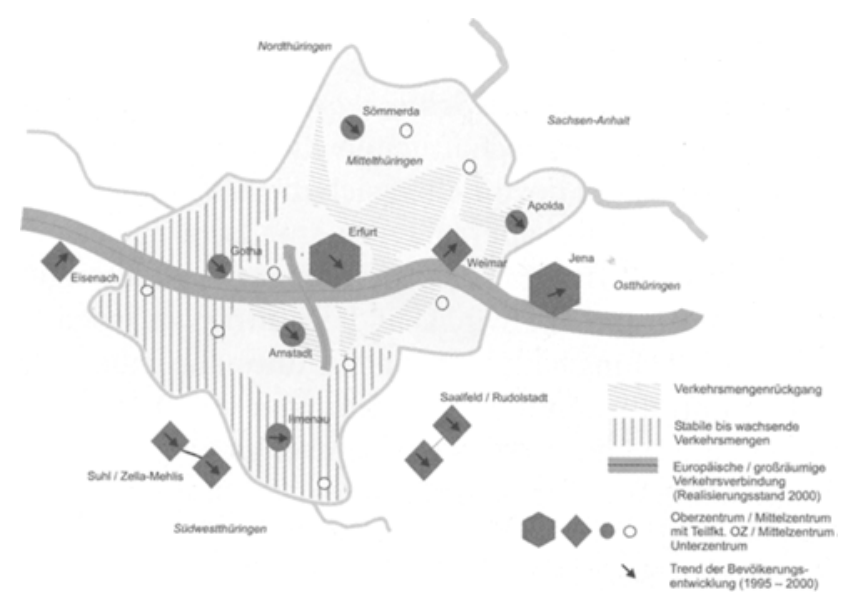

\subsection{Künftige Verkehrsmengenentwicklung}

Für die künftige Entwicklung der Verkehrsmengen in Mittelthüringen dürften wiederum die ökonomischen Rahmenbedingungen, der demographische Wandel, die Entwicklungen in der Raum- und Siedlungsstruktur sowie die Entwicklungen anderer Verkehrsträger und die steigende Pkw-Verfügbarkeit von (teilweise gegenläufigem) Einfluss sein:

- Im Bereich des motorisierten Individualverkehrs könnte vor allem im dünn besiedelten ländlichen Raum ein Verkehrsmengenrückgang wahrscheinlich werden (s. Abb. 2). Denn dort wird künftig die Bevölkerung stärker zurückgehen als bisher und die Überalterung der Bevölkerung am deutlichsten ausfallen; die großen Zentren werden dagegen geringere Bevölkerungsverluste erleiden. Nicht zu erwarten ist jedoch, dass die Verkehrsmengen proportional zum Bevölkerungsrückgang abnehmen, da die zurückzulegenden Wege für die verbleibenden Bewohner vor allem durch die Ausdünnung der Versorgungsinfrastruktur weiter werden und sich das Angebot im Straßenpersonennahverkehr ver- 
schlechtern wird. Da weder mit großen Steigerungen beim verfügbaren Einkommen noch mit einer wesentlichen Zunahme an Freizeit (außer durch die steigende Zahl an Rentnern ${ }^{9}$ ) zu rechnen ist, wird auf der anderen Seite auch der Freizeitverkehr den Trend zur insgesamt rückläufigen Verkehrsmengenentwicklung nicht kompensieren.

Wie sich der motorisierte Individualverkehr auf europäischen/großräumigen Verbindungen entwickeln wird, wird u. a. vom Geschäftsverkehr und damit von der ökonomischen Entwicklung abhängen. Diese ist jedoch besonders schwierig zu prognostizieren. Da hier jedoch zumindest der Bevölkerungsrückgang und die Überalterung keine so große Rolle spielen, dürfte zumindest nicht mit einer Verkehrsmengenabnahme zu rechnen sein.

- Die Entwicklung des Schwerlastverkehrs wird maßgeblich von der wirtschaftlichen Entwicklung beeinflusst. Ausgehend davon, dass sich die Wirtschaft zwar etwas erholen wird, aber keine Quantensprünge zu erwarten sind, dürfte er insgesamt höchstens geringfügig zunehmen. Räumlich wird sich die künftige wirtschaftliche Entwicklung hauptsächlich in Autobahnnähe vollziehen und dürften die Verflechtungen mit Osteuropa weiter zunehmen, so dass der Schwerlastverkehr wohl hauptsächlich auf den europäischen Verbindungen ansteigen wird.

Auch in der einschlägigen Fachliteratur ist von einer künftigen ungebremsten Verkehrsmengenexplosion auf der Straße nicht (mehr) unbedingt die Rede ${ }^{10}$; vielmehr wird "die noch immer von der Politik beschworene Vorstellung von einem außer Kontrolle geratenden Individualverkehr" als ,absurd" eingestuft. ${ }^{11}$ Aus heutiger Sicht ist sicherlich auch die Prognose des aktuellen Bundesverkehrswegeplans in Frage zu stellen, die noch 2000 unter anderem von einer „absehbaren Beruhigung der Kraftstoffpreisentwicklung“, von einem jahresdurchschnittlichen Wachstum des Bruttoinlandprodukts von 2,1\% zwischen 1997 und 2015 und von "steigenden verfügbaren Einkommen" ausging. Dem entsprechend wurde die Tatsache, dass die Verkehrsleistung des MIV im Jahr 2000 gegenüber 1999 um 3 \% zurückging, nur als eine kurzfristige Delle in einer sonst dauerhaften Entwicklung gewertet. ${ }^{12}$ Zumindest in den Jahren 2001 und 2002 war die Tendenz aber wohl weiterhin rückläufig. ${ }^{13}$

Der Wissenschaftliche Beirat beim Bundesministerium für Verkehr, Bau- und Wohnungswesen gelangte zu der Auffassung, dass mittelfristig eine gedämpfte weitere Zunahme von Verkehrsaufkommen und Verkehrsleistungen, langfristig aber eine Abnahme von Personenverkehrsaufkommen und Personenverkehrsleistungen zu erwarten seien. Gleichzeitig wird diese Aussage aber relativiert und darauf verwiesen, dass der demographische Wandel bezogen auf ganz Deutschland zwar eher lang- oder allenfalls mittelfristig spürbar werde, ,teilräumlich, $d$. h. bezogen auf einzelne Bundesländer, auf einzelne Regionen, (...) starke Veränderungen jedoch nicht erst mittelfristig, sondern sogar kurzfristig zu erwarten" sind. ${ }^{14}$

\section{Abbildung 2}

Schematische Darstellung eines Szenarios „Straßenverkehr“ für die Region Mittelthüringen.

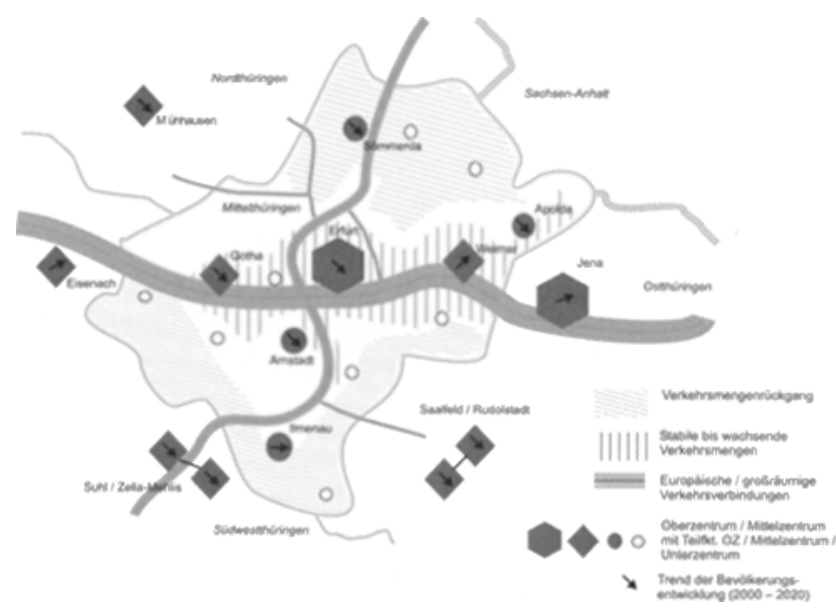

\section{Schlussfolgerungen für die Fortschreibung Funktionaler Straßennetze}

\section{1 Überlegungen zur künftigen Bedeutung und Wirkung von Funktionalen Straßennetzen}

Bevor Schlussfolgerungen für die Gestaltung von Funktionalen Straßennetzen gezogen werden lohnt es, sich am Beispiel Mittelthüringen die Zielsetzungen dieses Instruments zu vergegenwärtigen. Laut Regionalem Raumordnungsplan Mittelthüringen soll das Funktionale Straßennetz die dezentrale Konzentration unterstützen - zum einen durch Gewährleistung der Erreichbarkeit zentralörtlicher Einrichtungen aus den Versorgungsbereichen, zum anderen durch Sicherstellung wirtschaftlicher Entwicklungsmöglichkeiten in allen (Teil-)Räumen - und dadurch einen Beitrag zur Herstellung gleichwertiger Lebensverhältnisse leisten. ${ }^{15}$

Gerade das Postulat der gleichwertigen Lebensverhältnisse steht aber bekanntlich in letzter Zeit zumindest in Fachkreisen zur Diskussion ${ }^{16}$ - jedenfalls dann, wenn darunter zu verstehen ist, dass die Lebensverhältnisse über ganz Deutschland hinweg nach oben angeglichen 
werden sollen. In Zeiten wirtschaftlicher Flaute und desolater öffentlicher Haushalte fehlen für die dazu erforderliche Infrastrukturausstattung in weiten Teilen die finanziellen Mittel. Doch inwiefern wird oder sollte von Sparmaßnahmen auch die (Straßen-)Verkehrsinfrastruktur betroffen sein?

Zu bedenken ist, dass die (Straßen-)Verkehrsinfrastruktur ein sehr langlebiges und räumlich nicht verlagerbares Gut ist, für das auch nach seiner Herstellung laufend finanzielle Mittel bereitgestellt werden müssen. ${ }^{17}$ Nach Schätzungen des Deutschen Instituts für Wirtschaftsforschung müssten für den Zeitraum von 1990 bis 2020 zwei Drittel der geplanten Gesamtinvestitionen für die Bundesfernstraßen (129 Mrd. €) allein für den Ersatzbedarf veranschlagt werden. ${ }^{18}$ Diese Kosten werden sich bei einer sinkenden Bevölkerungszahl und wachsenden Überalterung auf immer weniger Steuerzahler verteilen. Bereits heute lauten die Schlagzeilen in Thüringer Zeitungen: „Geld reicht nur zum Löcher flicken - Zustand der Straßen in Thüringen wird immer schlechter". ${ }^{19}$ In welchem Umfang es unter diesen Bedingungen möglich und sinnvoll sein wird, künftig die Verkehrsinfrastruktur weiterhin auszubauen, erscheint fraglich.

Ist es also angebracht, künftig die regionalplanerische Basis, auf der Festsetzungen zum Funktionalen Straßennetz getroffen werden, um weitere Gesichtspunkte zu ergänzen? Bislang wurden in Thüringen die Funktionalen Straßennetze der Regionalen Raumordnungspläne auf der Grundlage des Landesentwicklungsprogramms, des Zentrale-Orte-Systems und der sich daraus ergebenden Verbindungserfordernisse erarbeitet und bei ergänzenden Festsetzungen zur Netzgestaltung gegebenenfalls die aktuellen Verkehrsmengen berücksichtigt. Muss sich der Planer künftig zudem eingehend mit der Frage beschäftigen, wie sich die Verkehrsmengen auf den einzelnen Strecken voraussichtlich entwickeln werden, damit die Raumordnung keinen Beitrag dazu leistet, dass mittel- bis langfristig womöglich nicht benötigte und auch nicht bezahlbare Netzelemente entstehen?

Auf der anderen Seite: Muss nicht gerade heute und in Zukunft die Verkehrsinfrastruktur eine solche Qualität aufweisen, dass sie unter den Vorzeichen einer Ausdünnung und Konzentration von Infrastruktureinrichtungen deren Erreichbarkeit ausreichend gewährleisten kann? Oder ist es finanziell gesehen doch besser und nicht zuletzt bürgerfreundlicher, z. B. die Einrichtungen der sozialen Infrastruktur begrenzt zu konzentrieren und dafür Abstriche bei der Verkehrsinfrastruktur hinzunehmen? Beides würde in unterschiedlicher Weise vordergründig zu einer unvermeidlichen Einschränkung der Mobilität führen. Auf längere Sicht jedoch wird sich unter den gegebenen Bedingungen nur durch Prioritäten - zunächst einmal unabhängig davon, wie diese gesetzt werden - ein Höchstmaß an Mobilität für möglichst große Bevölkerungskreise gewährleisten lassen.

Angesichts des Dilemmas, aus finanziellen Gründen wohl entweder die eine oder die andere Alternative verfolgen zu müssen, sollte eine politische Richtungsentscheidung getroffen werden, auch wenn dann im Einzelfall den spezifischen Umständen immer noch große Bedeutung zukommen dürfte. Die Raumordnung sollte sich aktiv in einen solchen Diskussionsprozess einbringen oder diesen gar erst in Gang setzen. Schließlich ist sie dadurch, dass ihr Zentrale-Orte-System und die Funktionalnetze steuernd auf diese Bereiche einwirken (sollten), bei der Fortschreibung ihrer Pläne direkt von diesen Fragestellungen betroffen.

Wie aber sieht es mit der raumordnerischen Zielsetzung aus, mittels des Funktionalen Straßennetzes zu wirtschaftlichen Entwicklungsmöglichkeiten in allen Teilräumen beizutragen? Sowohl in der Fachdiskussion als auch zunehmend in der politischen Debatte wird vermehrt die Position vertreten, Investitionen (und Subventionen) nicht mehr nach dem „Gießkannenprinzip“ zu verteilen, sondern sich vielmehr auf einwohnerstärkere Standorte mit guten Entwicklungsvoraussetzungen zu konzentrieren. Gegenüber dem regionalen Ausgleichsziel gewinnen damit die neue ökonomische Geographie sowie Wachstumstheorien an Bedeutung. Könnten und sollten von diesen Überlegungen auch Investitionen in die Verkehrsinfrastruktur erfasst werden?

Während über Jahre hinweg der Verkehrsinfrastruktur praktisch eine herausragende Bedeutung als Voraussetzung für wirtschaftliche Entwicklung beigemessen wurde, scheinen sich in letzter Zeit zweifelnde Stimmen zu mehren. So kommt eine Studie zu den regionalen Auswirkungen europäischer Verkehrspolitik zu dem Ergebnis, dass „die Auswirkungen von Investitionen in die Verkehrsinfrastruktur auf die Wirtschaftsentwicklung der Regionen (...) gering zu sein“ scheinen und "selbst starke Verbesserungen der regionalen Erreichbarkeit $(. .$.$) sich nur in geringen Steigerungen der re-$ gionalen Wirtschaftsaktivität" niederschlagen. ${ }^{20}$ An anderer Stelle wird betont, dass „Verkehrsinfrastruktur (...) allenfalls eine notwendige, aber keine hinreichende Erklärungsgröße für (regionales) wirtschaftliches Wachstum" ist, sondern insbesondere im ländlichen Raum von weiteren Investitionen begleitet sein muss. ${ }^{21}$ Verschiedentlich wird sogar festgestellt, dass „mit der Inbetriebnahme von Verkehrsinfrastruktur (...) für die betroffenen Regionen dennoch durchaus per Saldo negative (räumlich entleerende) statt positive Wirkungen 
entstehen können." 22 So wird z.B. in Thüringen die Inbetriebnahme der A71 von Erfurt bis Meiningen als Erklärung für den Kaufkraftabfluss der Stadt Ilmenau an Erfurt herangezogen. ${ }^{23}$

Damit wird deutlich, dass es offensichtlich keinen sachlich gerechtfertigen Grund gibt, die Verkehrsinfrastruktur grundsätzlich aus der Diskussion um die Konzentration von Maßnahmen und Investitionen zur Förderung der wirtschaftlichen Entwicklung auszunehmen - zumal es speziell in Thüringen spätestens nach der Fertigstellung der bereits begonnenen bzw. in Planung befindlichen Fernstraßen(aus)bauvorhaben keine Teilräume mehr geben wird, die überregional / großräumig wirklich problematisch zu erreichen wären.

\subsection{Vorschläge für die künftige Gestaltung eines Funktionalen Straßennetzes}

Im Ergebnis der angestellten Überlegungen wird vorgeschlagen, auch künftig zunächst das Zentrale-OrteSystem als Bezugspunkt für die Ausweisung der Funktionalnetzebenen heranzuziehen. Dies dient zum einen der Nachvollziehbarkeit der Einstufungen. Zum anderen haben die obigen Überlegungen gezeigt, welch enger Zusammenhang zwischen beiden Instrumenten besteht.

Den Funktionalnetzebenen zugeordnete textliche Festsetzungen können dazu herangezogen werden darzustellen, welche Zielsetzungen mit der jeweiligen Funktionalnetzebene verfolgt werden, und ebenso, welche grundsätzlichen Konsequenzen sich daraus für weitere Investitionen ergeben (sollten). An diesem Punkt schließlich sollten auch absehbare Entwicklungen der Verkehrsmengen Berücksichtigung finden, hat doch die Analyse gezeigt, dass nicht mehr pauschal von einer Zunahme ausgegangen werden kann. Statt also, wie zumindest in Mittelthüringen bislang geschehen, im großen Stil Ausbaumaßnahmen für alle Ebenen des Funktionalnetzes zu fordern, müsste die Raumordnung nun differenzierter agieren und z. B. nach Funktionalnetzebenen unterscheiden, deren Verbindungen

- eines weiteren Ausbaus bedürfen,

- zwar keines weiteren Ausbaus bedürfen, aber vorrangig in einem guten Zustand zu erhalten sind,

- bei vorhandenen finanziellen Mitteln in einem guten Zustand erhalten werden sollen.
Eine ausreichende Diskussion über den Zusammenhang zwischen Zentrale-Orte-System und Funktionalnetzen hat bislang in Thüringen nicht stattgefunden. Vielmehr wurden beide Instrumente bei der Erstellung des Landesentwicklungsplans (LEP) von vornherein faktisch weitgehend voneinander abgekoppelt. ${ }^{24}$

Für die Regionalplanung ist die weitere Ausgestaltung des Funktionalnetzes insofern festgelegt, als der LEP vorgibt, dass künftig keine Unter- und Kleinzentren, sondern Grundzentren auszuweisen sind, die wiederum bestimmte Mindestanforderungen bezüglich der Einwohnerzahlen erfüllen müssen. Da begründete Ausnahmen möglich sind, haben die Regionen dennoch einen gewissen Spielraum für eine Diskussion darüber, wie weit sie den Konzentrationsprozess treiben wollen. In Mittelthüringen wird nach dem derzeitigen Stand des Entwurfsprozesses der Ansatz verfolgt, nur in wenigen Ausnahmen zusätzliche Grundzentren auszuweisen, so dass es zu einer deutlichen Ausdünnung der Zentralen Orte unterer Stufe kommen wird. Dennoch wird aus nahezu jedem Ort der Region in einer Entfernung von $15 \mathrm{~km}$ (Straßenlänge) bzw. innerhalb von 30 Minuten Fahrzeit mit dem ÖPNV ein Grundzentrum (bzw. Mittel- oder Oberzentrum) zu erreichen sein. Da die Koppelung der Funktionalnetze mit dem ZentraleOrte-System im fortzuschreibenden Regionalplan beibehalten werden soll, bedeutet dies, dass zahlreiche der bislang ausgewiesenen kleinräumigen Verbindungen wegfallen werden.

Die zum Funktionalen Straßennetz gehörigen textlichen Festsetzungen finden sich zum Teil bereits im LEP oder sind durch diesen in ihrer Richtung vorgegeben. ${ }^{25}$ Demnach gelten für die einzelnen Straßenverbindungen folgende Zielsetzungen:

- Europäisch bedeutsame Straßenverbindungen:

Übergeordnete Zielsetzung:

Verbindungen zwischen Oberzentren und Metropolräumen, Verbesserung der Erreichbarkeit des Landes im Güter- und Personenverkehr

Konkrete Zielsetzung:

Vordringlicher Ausbau

- Großräumig bedeutsame Straßenverbindungen:

Übergeordnete Zielsetzung:

Anbindung der Mittelzentren mit Teilfunktionen eines Oberzentrums an die europäische Netzebene

Konkrete Zielsetzung:

Schrittweise Maßnahmen zur Erhöhung der Leistungsfähigkeit und der möglichen Reisegeschwindigkeit, abschnittweiser drei- oder vierspuriger Ausbau, Bau von Ortsumgehungen 
- Überregional bedeutsame Straßenverbindungen:

Übergeordnete Zielsetzung:

Verbindungen zwischen den Mittelzentren mit Teilfunktionen eines Oberzentrums, Anbindung der Mittelzentren an die übergeordnete Netzebene, Sicherung der gleichwertigen Entwicklung aller Landesteile

Konkrete Zielsetzung:

Ausbau einzelner konkret benannter Verbindungen

- Regional bedeutsame Straßenverbindungen:

Übergeordnete Zielsetzung:

Verbindungen benachbarter Mittelzentren, Anbindung der Grundzentren an die Ober- und Mittelzentren, Verbindung der Grundzentren untereinander

Konkrete Zielsetzung:

Aufgabe des Regionalplans.

Vor dem Hintergrund der Ergebnisse der Verkehrsmengenanalyse und der Verkehrsmengenabschätzung müssen die im LEP getroffenen Festsetzungen unterschiedlich bewertet werden:

Den Zielsetzungen für die europäische Ebene kann uneingeschränkt zugestimmt werden. Die Analyse hat gezeigt, dass auf diesen Verbindungen mit einem steigenden Verkehrsaufkommen zu rechnen ist.

Auf den Verbindungen der großräumigen Ebene dürfte der Verkehrsmengenzuwachs höchstens moderat ausfallen, da der hierfür nach der Abschätzung hauptsächlich verantwortliche Lkw-Anteil geringer ist. Damit könnten ergänzende Festsetzungen im Regionalplan sinnvoll werden, die entweder für alle Ausbaumaßnahmen an großräumigen Verbindungen eine Orientierung an den mittel- bis langfristig zu erwartenden Verkehrsmengen fordern oder aber ggf. erforderliche Ausbaumaßnahmen direkt benennen.

Die Verbindungen der überregionalen Ebene haben in der Verkehrsmengenanalyse ein sehr heterogenes Bild abgegeben - einige wiesen Verkehrsmengenzuwächse zwischen 1995 und 2000 auf, einige aber auch einen Rückgang. Dementsprechend ist es grundsätzlich sinnvoll, dass der LEP nur für ausgewählte Verbindungen ausdrücklich einen Ausbau fordert.

Da für die meisten der im LEP ausgewiesenen überregionalen Verbindungen keine textlichen Festsetzungen getroffen wurden, sind zudem ergänzende Regelungen im Regionalplan sinnvoll, die die restlichen Verbindungen gemäß der zu erwartenden Verkehrsmengen z. B. in "überregionale Verbindungen mit Ausbaubedarf" und "überregionale Verbindungen mit Sicherungsbedarf" (gleich vorrangige Unterhaltung) einordnen.
Die regional bedeutsamen Verbindungen haben nach den Ergebnissen der Verkehrsmengenanalyse für die Jahre 1995 bis 2000 eine ebenso heterogene Entwicklung erfahren wie die überregionalen Verbindungen. Vor allem der MIV hat auf vielen Verbindungen proportional deutlich zugenommen. Hieraus jedoch einen Ausbaubedarf abzuleiten wäre vorschnell. Ein Blick auf die Verkehrsmengen im Jahr 2000 zeigt, dass die Verkehrsbelastung in den meisten Fällen immer noch recht gering ist, so dass das Verkehrsmengenwachstum in der Regel von einem sehr niedrigen Niveau aus erfolgt. Die textlichen Festsetzungen im Regionalplan sollten daher einen Ausbau nur in Ausnahmefällen vorsehen, ansonsten aber mit Hinweis auf die Ausdünnung bei den Grundzentren zumindest eine vorrangige Unterhaltung dieser Netzstufe fordern.

Darüber hinaus könnte vorgesehen werden, sonstige, keiner Netzebene zugeordnete Straßen, die aus dem Grundversorgungsbereich unmittelbar auf das Grundzentrum zulaufen, bei Vorhandensein der finanziellen Mittel nachrangig zu den regionalen Verbindungen, aber vorrangig vor anderen Straßen zu unterhalten.

\subsection{Vorschlag für den weiteren Umgang mit bestehenden Funktionalen Straßennetzen}

Abschließend stellt sich die Frage, wie in Regionen mit Bevölkerungsrückgang bis zum Inkrafttreten der neuen Festsetzungen aus landes- und regionalplanerischer Sicht mit Straßenbauvorhaben umgegangen werden soll. So gibt es z. B. in Thüringen derzeit keine Festsetzung, die eine Orientierung der Straßenbauvorhaben am Bedarf fordert, vielmehr wird grundsätzlich für alle Netzebenen der Ausbau der Straßen angestrebt. Dennoch sollten vor allem die Raumordnugsverfahren durchführende Landesplanungsbehörde, aber auch die Regionalplanung selbst, die im Rahmen solcher Verfahren regelmäßig eine Stellungnahme abgibt, verstärkt ihr Augenmerk auf die Verkehrsmengenprognosen richten. Denn das Raumordnungsverfahren dient dazu, die Raumverträglichkeit einer Planung oder Maßnahme zu prüfen, und es erscheint nur konsequent, bei solchen Straßenbauvorhaben, die den mittelfristigen Bedarf überschreiten, die Raumverträglichkeit im Hinblick auf die meist zahlreichen konkurrierenden Belange in Frage zu stellen. Wenigstens sollte in solchen Fällen ggf. versucht werden, zusammen mit der Fachplanung statt der häufig beantragten "großen Lösungen" unter Ausnutzung der in den einschlägigen „Richtlinien für die Anlage von Straßen" eröffneten Spielräume zu kleineren, verträglicheren Alternativen zu gelangen.

Zumindest in Thüringen sind Verkehrsmengenprognosen bereits heute fester Bestandteil der Anhörungs- 
unterlagen zu Straßenbauvorhaben. Allerdings sind sie nur zur Information der Beteiligten gedacht und flieBen nicht in die raumordnerische Bewertung mit ein. Für eine kritische Auseinandersetzung mit den Zahlen müsste - anders als bisher - auch der Kern der Prognose, nämlich die Annahmen, auf denen sie beruhen (Bevölkerungsentwicklung, wirtschaftliche Entwicklung, usw.), preisgegeben werden. ${ }^{26}$ Hier sollte die obere Landesplanungsbehörde vom Vorhabenträger eine angemessene Darlegung einfordern.

\section{Fazit}

Bislang war die Sache einfach: Wenn vom Straßenverkehr die Rede war, wurde eigentlich immer von einer bisher und auch künftig steigenden Verkehrsmenge ausgegangen.

Die Analyse der Verkehrsmengen in Mittelthüringen hat jedoch gezeigt, dass pauschale Aussagen zur Verkehrsmengenentwicklung heute nicht mehr möglich sind - nicht für ganze Regionen oder Länder und schon gar nicht für Gesamtdeutschland. ${ }^{27}$ Tatsächlich ist es wohl so, dass der Straßenverkehr in Teilräumen bereits eine Sättigungsgrenze erreicht hat oder mittelfristig erreichen wird. Dieser Erkenntnis sollte sich die Raumordnung insbesondere in den vom Bevölkerungsrückgang betroffenen Räumen nicht verschließen. Im Gegenteil: Der Empfehlung des Wissenschaftlichen Beirats für Verkehr beim Bundesministerium für Verkehr, Bau und Wohnungswesen, „die demographischen Veränderungen verstärkt zur Grundlage der Ausgestaltung von Verkehrspolitik, der Entscheidungen über Verkehrsinfrastrukturen, Verkehrsangebote und Verkehrsmanagement $\mathrm{zu}$ machen ${ }^{28}$, ist auch für die Raumordnung zuzustimmen. Zwar ist es nicht zweckdienlich, erwartbare Verkehrsmengen gleich zur Grundlage für die Gliederung der Funktionalnetze zu erheben - der querschnittsorientierte Ansatz der Raumordnung stellt hier aus gutem Grunde andere Anforderungen. Die Verkehrsmengenentwicklung kann und muss bei der Formulierung von detaillierten Zielsetzungen für die einzelnen Netzstufen jedoch Berücksichtigung finden.

Die Raumordnung sollte sich folglich dieses Themas annehmen, mehr noch: Sie sollte auch im Bereich des Straßenverkehrs eine Diskussion zu den Auswirkungen des Bevölkerungsrückgangs und den erforderlichen Anpassungsstrategien anschieben - auch wenn diese Punkte vermutlich noch schwieriger als auf anderen Sachgebieten zu vermitteln sein dürften, nachdem jahrelang sowohl von der Fachplanung als auch von den politischen Entscheidungsträgern stets nur auf immer mehr und noch breitere Straßen hingearbeitet wurde.

\section{Anmerkungen}

(1)

Vgl. z.B.: Topp, Hartmut: Stadt- und Regionalverkehr 2030. In: Der Nahverkehr, Heft 7-8/2004, S. 34 ff.

(2) Regionaler Raumordnungsplan Mittelthüringen; veröff. als Sonderdruck des Thür. Staatsanzeigers Nr. 40/99 vom 4. Oktober 1999 (als Download im Internet unter www.regionalplanung.thueringen.de verfügbar, jedoch ohne die thematischen Karten z.B. zu den Funktionalnetzen)

(3) Richtlinie für die Anlage von Straßen. Teil: Leitfaden für die funktionale Gliederung des Straßennetzes

(4)

Der Landesentwicklungsplan Thüringen wurde am 30.10.2004 als Rechtsverordnung verbindlich und ist als Thüringer Verordnung über den Landesentwicklungsplan vom 06.10.2004 im Gesetzund Verordnungsblatt Thüringen (GVBl. S. 754 ff.) veröffentlicht. Er ist als Download im Internet unter: www.thueringen.de/de/ tmbv/rolp/ verfügbar.

(5)

Die Verkehrszählungen finden an fünf bis sechs Tagen - darunter Werktage, Sonn- und Feiertage sowie Ferientage - über das Jahr verteilt statt, werden auf das ganze Jahr hochgerechnet (mit einem kleinen methodischen Unterschied zwischen den Jahren 1995 und 2000) und dann als durchschnittlicher täglicher Verkehr umgelegt. Neben den temporären Zählstellen gibt es einige Dauerzählstellen, die zur Eichung des Systems dienen.

(6)

Regionale Straßenverbindungen entsprechen dem Vorentwurf zum Regionalplan der Regionalen Planungsstelle Mittelthüringen. Rundungsfehler sind nicht ausgeglichen. Verbindungen ohne Kategorisierung im Stadtgebiet werden der Vollständigkeit halber aufgeführt, bleiben aber in Bezug auf Schlussfolgerungen für das künftige Funktionalnetz ohne Bedeutung.

(7)

Regionale Planungsgemeinschaft Nordthüringen (2004): Pendlerverflechtungen in Nordthüringen. Sondershausen, Anlage 4. Dagegen sind die Pendlerverflechtungen nach Niedersachsen und Hessen im selben Zeitraum stark angestiegen.

(8)

Die Standardabweichung gibt das Maß für die Streuung um den Mittelwert an. Je höher der Wert, desto größere Abweichungen vom Mittelwert finden sich unter den Daten. Die relativ homogene Entwicklung der einzelnen Kategorien ist Voraussetzung für planerische Zielaussagen bezüglich des Ausbau- und Erhaltungszustands der Funktionalnetzkategorien.

(9)

Einerseits kann eine größere, mobile und zudem autoaffin geprägte zukünftige Rentnergeneration mehr Verkehr erzeugen als bisherige Rentnergenerationen, sie wird aber andererseits durch Freizeitverkehr nicht die Verkehrsmengen generieren, die sie zuvor 
im Arbeitsleben erbracht hat. Nach der KONTIV-Erhebung (Kontinuierliche Erhebung zum Verkehrsverhalten) des BMVBW war die durchschnittliche von Rentnern zurückgelegte Tagesdistanz im Jahre 2002 weniger als halb so hoch wie die von Erwerbstätigen. Vgl. Holz-Rau, Christian und Scheiner, Joachim:Verkehrsplanung und Mobilität im Kontext der demographischen Entwicklung. In: Straßenverkehrstechnik, Heft 7/2004, S. 344.

(10)

Vgl. z.B. Chlond, B.; Manz, W.; Zumkeller, D.: Stagnation der Verkehrsnachfrage - Sättigung oder Episode? In: Internationales Verkehrswesen, Heft 9/2002, S. 396 ff.

(11)

Diekmann, Achim: Verkehrspolitik unter falschen prognostischen Prämissen. In: Internationales Verkehrswesen, Heft 3/1998, S. 81

(12)

Vgl. Gresser, Klaus et al: Verkehrsprognose 2015 für die Bundesverkehrswegeplanung. In: Internationales Verkehrswesen, Heft $12 / 2001$, S. $585 \mathrm{ff}$.

(13)

Vgl. Bundesministerium für Verkehr, Bau- und Wohnungswesen (Hrsg.): Verkehr in Zahlen 2003/2004, Hamburg, 2003, S. 159

(14)

Demographische Veränderungen - Konsequenzen für Verkehrsinfrastrukturen und Verkehrsangebote. Papier des Wissenschaftlichen Beirats beim Bundesminister für Verkehr, Bau- und Wohnungswesen, Stand: 26. Januar 2004, abgedruckt in: Informationen zur Raumentwicklung, Heft 6/2004, Anhang 1, S. 401 ff.

(15)

Regionaler Raumordnungsplan Mittelthüringen vom 04.Oktober 1999, Begründung zur Festsetzung 9.4.4.

(16)

Vgl. z.B. in Gatzweiler, Hans-Peter; Kocks, Martina: Demographischer Wandel - Modellvorhaben der Raumordnung als Handlungsfeld des Bundes. In: Raumforschung und Raumordnung, Heft 2/2004, S. 138

(17)

Vgl. Klatt, Sigurd: Verkehrsinfrastruktur, in: Akademie für Raumforschung und Landesplanung (Hrsg.): Handwörterbuch der Raumordnung, Hannover, 1995, S. 1017; vgl. Demographische Veränderungen - Konsequenzen für Verkehrsinfrastrukturen und Verkehrsangebote. Papier des Wissenschaftlichen Beirats beim Bundesminister für Verkehr, Bau- und Wohnungswesen, Stand: 26. Januar 2004, abgedruckt in: Informationen zur Raumentwicklung, Heft 6/2004, Anhang 1, S. 411

(18)

Vgl. Kunert, Uwe; Link, Heike: Bundesfernstraßen - enge Spielräume für Netzerweiterungen auch bei hohen Investitionen. In: Informationen zur Raumentwicklung, Heft 6/2004, S. 395

Thüringische Landeszeitung vom 21.06.2004, Ostthüringer Zeitung vom 27.07.2004
(20)

Schürmann, Carsten; Spiekermann, Klaus, Wegener, Michael: Transeuropäische Verkehrsnetze und regionale Entwicklung. In: RaumPlanung, Heft 116, 2004, S. 210

(21)

Blum, Ulrich: Raumwirtschaftliche Effizienz von Verkehrsinfrastrukturinvestitionen, in: Informationen zur Raumentwicklung, Heft 6/2004, S. 379 und 386

(22)

Sichelschmidt, Henning: Neue Bundesländer: Fortschritte bei der Verkehrsanbindung. In: Internationales Verkehrswesen, Heft $9 / 2004$, S. 384

(23)

Vgl. Kaufen in Ilmenau - gut wie nie zuvor: Großstadtniveau, in: Freies Wort vom 16.02.2005, S. 22

\section{(24)}

Zwar wird in der Begründung zum Ziel 4.1.6 LEP (Gliederung des funktionalen Verkehrsnetzes) das Zentrale-Orte-System zugrunde gelegt, doch folgt die zeichnerische Einstufung der Straßen in die einzelnen Verbindungskategorien dem nur eingeschränkt. Zudem hat sich die Abkopplung des Funktionalnetzes augenfällig darin gezeigt, dass trotz der weitreichenden Umstrukturierung der Zentralen Orte höherer Stufe infolge der Beteiligungsverfahren bei der Fortschreibung des LEP keine daraus resultierenden Änderungen im Funktionalnetz vorgenommen wurden.

\section{(25)}

Siehe Festsetzungen 4.1.6 bis 4.1.17 des LEP. Thüringer Verordnung über den Landesentwicklungsplan vom 06.10.2004, Gesetzund Verordnungsblatt Thüringen (GVBl. S. 754 ff.)

\section{(26)}

Vgl. Rommerskirchen, Stefan: Langfristige Verkehrsprognosen - Gratwanderungen in einem komplexen Problemgebirge. In: Internationales Verkehrswesen, Heft 7-8/1997, S. 365. Dort heißt es allgemein in Bezug auf den Umgang mit Verkehrsmengenprognosen: Explizit oder implizit getroffene Annahmen bei der Prognoseerstellung nicht zu dokumentieren, sei „eindeutig als unseriös zu bezeichnen und macht eine Prognose unbrauchbar“.

\section{(27)}

So sollen auch in einem vom BMVBW in Auftrag gegebenen Gutachten zu den langfristigen Auswirkungen des demografischen Wandels auf die Verkehrsentwicklung die Betrachtungen „räumlich disaggregiert erfolgen in der Erkenntnis, dass in Zukunft keine bundeseinheitlichen Entwicklungen mehr erfolgen, sondern die Entwicklungen in Teilräumen unterschiedlich verlaufen werden. “ in: Antwort der Bundesregierung auf die Große Anfrage "Reduzierung der zusätzlichen Flächennutzung für Verkehrs- und Siedlungszwecke“, Bundestags-Drucksache 15/4472 vom 06.12.2004, S. 30

(28)

Demographische Veränderungen - Konsequenzen für Verkehrsinfrastrukturen und Verkehrsangebote. Papier des Wissenschaftlichen Beirats beim Bundesminister für Verkehr, Bau- und Wohnungswesen, Stand: 26. Januar 2004, abgedruckt in: Informationen zur Raumentwicklung, Heft 6/2004, Anhang 1, S. 417 


\section{Literatur}

Blum, Ulrich: Raumwirtschaftliche Effizienz von Verkehrsinfrastrukturinvestitionen. Informationen z. Raumentwicklung (2004) H. 6, S. 379-388

Bundesministerium für Verkehr, Bau- und Wohnungswesen (Hrsg.): Verkehr in Zahlen 2003/2004. - Hamburg 2003

Chlond, B.; Manz, W.; Zumkeller, D.: Stagnation derVerkehrsnachfrage - Sättigung oder Episode? Internat. Verkehrswesen (2002) 9

Deutscher Bundestag (Hrsg.): Antwort der Bundesregierung auf die Große Anfrage „Reduzierung der zusätzlichen Flächennutzung fürVerkehrs- und Siedlungszwecke“. BT-Drucksache 15/4472 vom 06.12.2004

Diekmann, Achim:Verkehrspolitik unter falschen prognostischen Prämissen. Internat. Verkehrswesen (1998) 3, S. 76-81

Gatzweiler, Hans-Peter; Kocks, Martina: Demographischer Wandel - Modellvorhaben der Raumordnung als Handlungsfeld des Bundes. Raumforsch. u. Raumordnung (2004) H. 2, S. 133-148

Gresser, Klaus et al: Verkehrsprognose 2015 für die Bundesverkehrswegeplanung. Internat. Verkehrswesen (2001) 12, S. 585591

Holz-Rau, Christian; Scheiner, Joachim: Verkehrsplanung und Mobilität im Kontext der demographischen Entwicklung. StraBenverkehrstechnik (2004) H. 7, S. 341-348

Klatt, Sigurd: Verkehrsinfrastruktur. In: Handwörterbuch der Raumordnung. Hrsg.: ARL. - Hannover 1995, S. 1016-1023

Kunert, Uwe; Link, Heike: Bundesfernstraßen - enge Spielräume für Netzerweiterungen auch bei hohen Investitionen. Informationen z. Raumentwicklung (2004) H. 6, S. 395-400

Regionale Planungsgemeinschaft Nordthüringen: Pendlerverflechtungen in Nordthüringen. - Sondershausen 2004

Rommerskirchen, Stefan: Langfristige Verkehrsprognosen - Gratwanderungen in einem komplexen Problemgebirge. Internat. Verkehrswesen (1997) 7-8, S. 362-366
Schürmann, Carsten; Spiekermann, Klaus; Wegener, Michael: Transeuropäische Verkehrsnetze und regionale Entwicklung. RaumPlanung (2004) H. 116, S. 205-210

Sichelschmidt, Henning: Neue Bundesländer: Fortschritte bei der Verkehrsanbindung. Internat. Verkehrswesen (2004) 9, S. 379385

Thüringen, Landesamt für Straßenbau (Hrsg.): Verkehrsmengenkarte 1995. - Erfurt 1996

Thüringen, Landesamt für Straßenbau (Hrsg.): Verkehrsmengenkarte 2000. - Erfurt 2002

Thüringen, Ministerium für Bau und Verkehr (Hrsg.): Landesentwicklungsbericht 2004. - Erfurt 2004

Topp, Hartmut: Stadt- und Regionalverkehr 2030. Nahverkehr (2004) $7-8$, S. 34-39

Wissenschaftlicher Beirat beim Bundesminister für Verkehr, Bauund Wohnungswesen: Demographische Veränderungen - Konsequenzen für Verkehrsinfrastrukturen und Verkehrsangebote, Stand 26. Januar 2004. Abgedruckt in: Informationen z. Raumentwicklung (2004) H. 6, Anhang 1, S. 403-417

Regionaler Raumordnungsplan Mittelthüringen. Sonderdruck des Thüringer' Staatsanzeigers Nr. 40/99 vom 04.Oktober 1999 ( als Download unter www.regionalplanung.thueringen.de verfügbar, jedoch ohne die thematischen Karten z. B. zu den Funktionalnetzen)

Thüringer Verordnung über den Landesentwicklungsplan vom 06.10.2004. GVBl. Thüringen, S. 754 ff. (www.thueringen.de/de/ $\mathrm{tmbv} / \mathrm{rolp} /$ )

Dipl.-Geogr. Lars Liebe

Dipl.-Ing. Ute Weiß

Thüringer Landesverwaltungsamt

Regionale Planungsstelle Mittelthüringen

Weimarplatz 4

99423 Weimar

E-Mail: lars.liebe@tlvwa.thueringen.de ute.weiss@tlvwa.thueringen.de

\section{Hinweis:}

